\title{
Implementation of Low Power Multi-hop Wireless Sensor Network
}

\author{
Zhou Jianhong ${ }^{1,2,}$ Ma Maode ${ }^{1}$, Fengyong ${ }^{2}$ \\ ${ }^{1}$ Chengdu Information Technology of Chinese Academy of Science Co. Ltd, Chengdu Sichuan 610041, China \\ ${ }^{2}$ Nanyang Technological University, School of Electrical \& Electronics Engineering, 639798, Singapore
}

\begin{abstract}
In this paper, the multi-hop wireless sensor network is created, which is realized by Micaz and Mib520 Board using TinyOS and Cygwin. The mote transmission mode is engaged and off-the-shelf protocols and algorithm, RSSI and PDR, are applied in this wireless network and the performance is improved. Several experiments are proposed to set standard RSSI threshold value to allow the user to send packet with efficient power level.
\end{abstract}

Keywords: Wireless Sensor Network; RSSI; PDR; threshold value; Transmission Power

\section{Introduction}

Wireless networks technology has been consistently improving with time and increasingly finding its way into all aspects of people's daily lives. Medical application is a key field where Wireless Networking has a promising future. In the healthcare field, access and cost saving are two of the hottest issues these days. Wireless Technologies can contribute towards helping with both of these issues which is in great demand [1].

Negligent mistakes by doctors and nurses costs hospitals, insurance companies and the government a large sum of money each year. These costs can be reduced by reducing the number of mistakes. Efficient and secure data handling and resource management is another area where wireless networks can help. Deploying huge machines around the hospital can be very expensive and time consuming. With Wireless Technology interfaces can be designed such that access to the machines can be provided from anywhere in the hospital. This allows for rapid and flexible deployment. By increasing the doctors' and nurses' efficiency, hospitals can provide care for more patients and increase their profits. On the other hand, Power efficiency is also a key factor for every wireless sensor network. Recently, Daniel Morrison et al. has a prototype design of a reconfigurable, low cost and low power miniaturized sensor node that interfaces with a variety of sensors, logs and transmits data wirelessly.[2] Meanwhile, a cluster based routing algorithm[3] has been proposed for WSN to minimize the power consumption. In this paper, a reliable transmission of physiological data from ICU gateway to central server wirelessly in hospital environments was implemented by Crossbow products. Based on the analysis on the relation between message signal strength and transmission power, the off-the-shelf protocols and algorithms for TinyOs was applied and the performance was improved. At last, some possible methods were proposed to extend the usage of the battery energy.

\section{Hardware And Software Review}

\subsection{Micaz}

The Micaz mote platform is a third generation device used for enabling low-power, wireless sensor networks available in $2.4 \mathrm{GHz}$ and $868 / 916 \mathrm{MHz}$. The Micaz Mote offers a $2.4 \mathrm{GHz}$, IEEE/ZigBee 802. The MICA Mote platforms are fully compatible with the Mote Works Software Platform and enable users to set up ad-hoc [4]. However, there're two improvements applying on the traditional Micaz mote platform in our system as shown in Fig. 1 and Fig. 2. The rechargeable battery is being used in order to decrease the total size of Micaz. Furthermore, the original antenna is replaced with a flat one which looks more professional and practical. From the experiment's result, the USB connector of MIB520 board will influence the packet delivery ratio with very high percentage, so a flat cable is designed to solve this problem. The Base Station and MIB520 board will be connected through the flat cable. 


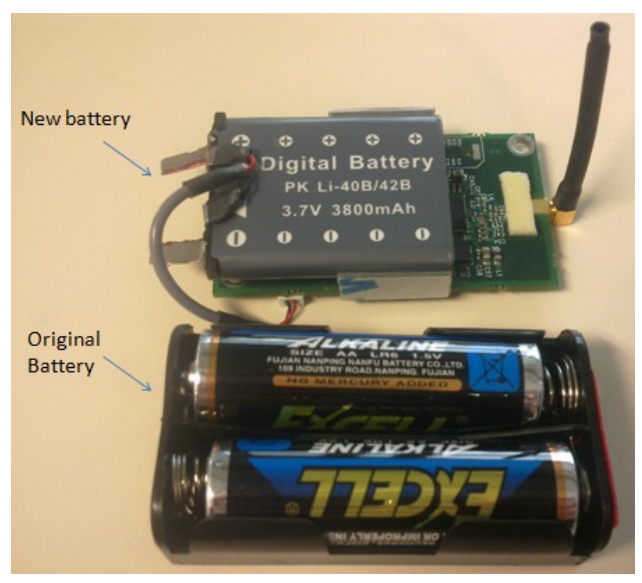

Fig. 1 Rechargeable Battery

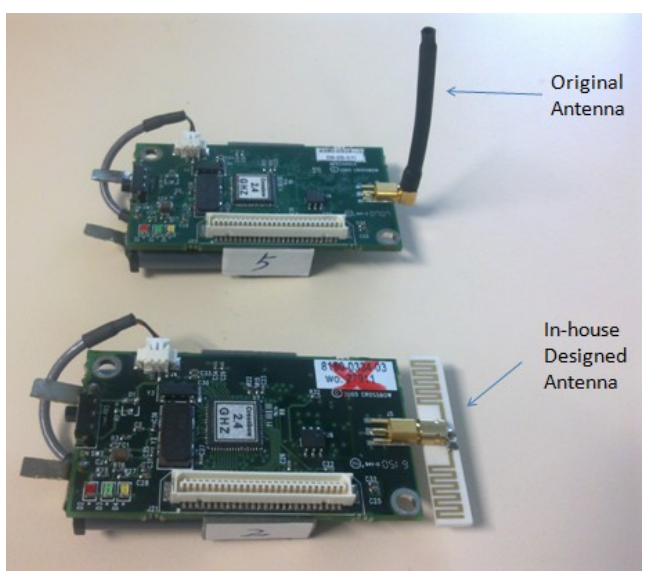

Fig. 2 In-house Designed Antenna

\subsection{Mib520 Board}

The MIB520CA provides USB connectivity to the MICA family of Motes for communication and in-system programming with attached 51 pins. Any MICAz node can be programmed as a base station when mated to the MIB520 USB interface board. In addition to data transfer, the MIB520 can also provide a USB programming interface.

\subsection{TinyOS}

TinyOS is a free and open source component-based operating system and platform targeting wireless sensor networks (WSN)[5]. TinyOS is an embedded operating system written in the nesC programming language as a set of cooperating tasks and processes. TinyOS programs are built out of software components, some of which present hardware abstractions. Components are connected to each other using interfaces. TinyOS provides interfaces and components for common abstractions such as packet communication, routing, sensing, actuation and storage[6].

\subsection{Cygwin}

It is free software that provides a Unix-like environment and software tool set to users of any modern version of MS-Windows for x86 CPUs (NT/2000/XP/Vista/7) and (using an older version of Cygwin) some obsolete versions (95/98/ME) as well. Cygwin consists of a UNIX system call emulation library, cygwin1.dll, together with a vast set of GNU and other free software applications organized into a large number of optional packages. Among these packages are high-quality compilers and other software development tools, a complete X11 development toolkit, GNU emacs, TeX and LaTeX, OpenSSH (client and server), and much more, including everything needed to compile and use PhysioToolkit software under MS-Windows[7].

Basically, the Micaz mote can be programmed and preconfigured by using the Cygwin interface under Microsoft environment. To figure out the best choice for a suitable power in order to save the battery energy, the maximum transmission range will be recorded. The Micaz mote is using the chip CC2420 which power index ranges from 1(Min.) to 31(Max.).

\section{Protocol}

\subsection{Mote Transmission}

TinyOS provides a number of interfaces to abstract the underlying communications services and a number of components that provide (implement) these interfaces. For demonstration purpose, two motes are being used to show how they can connect with each other. First of all, TinyOS library provides some very useful resource indicating some specific functions. For instance, the user can install two different programs into two Micaz mote named base-station and sending-mote separately. And then connect the base-station to PC through Mib520 programming board. The real code is designed and rebuilt based on the open source code provided by TinyOS Library, it allows user to define the program independently based on the specific requirement.

\subsection{RSSI vs PDR Protocol}

Each sending mote will send out 2000 packets to basestation with specific RSSI (Radio signal strengthen indication) value. Both of the RSSI value and Node_id (the node number of the mote which is used as a unique id for the mote tell the user where the received packet comes from) is assigned into the packet which can be sent to base-station, the system will help to create a file including these data in one default folder. Then the basestation can read the information by using the following printf command [8].

"printf("From node is \%d It RssiValue is \%d It seq num \%d lt pkt rcv \%d In ",rms->nodeid,rms$>$ RssiValue,rms->seqnum,counter); //rms->Tx);"

The RSSI vs PDR protocol builds and maintains the packet sending interval. The total number of packet can be pre-set from the code [9].

To compile the program of Micaz mote, basically it needs four files in the source folder: Makefile, Header file, Configuration file and Application file. The transmission power index, channel number and packet sending time interval can be set directly from Makefile. 
One of the goals is to estimate the most efficient transmission power for each specific link, and it can be determined by setting the RSSI (radio signal strength indication) threshold value in order to keep the PDR (packet delivery ratio) higher than $90 \%$.

For power control protocol, the basic procedures consists three steps. Firstly, the sending mote sends packet to base-station. The packet contains RSSI value, Node_id and other useful information. Then, the base-station will transmit these information to the sending mote again which is going to be compared with the standard RSSI threshold value. Finally, the sending mote will adjust its transmission power level based on these information.

\section{Experiment and Discussions}

\subsection{Experiment Procedures}

In order to avoid any unexpected influence, all the experiment including free space and on-body test is done in the chamber where can provide almost perfect environment and lowest impacts. The experiment procedure will be as following steps.

1. Nodes should be programmed as Base station and Mote, respectively.

2. The base station is connected to the work station through the programming board. It will receive packet and read the information attached.

3. Check the port number of Mib520 board from 'Device Manager' in 'My Computer' shown as Fig. 3. Need to check every time when connect it to the computer.

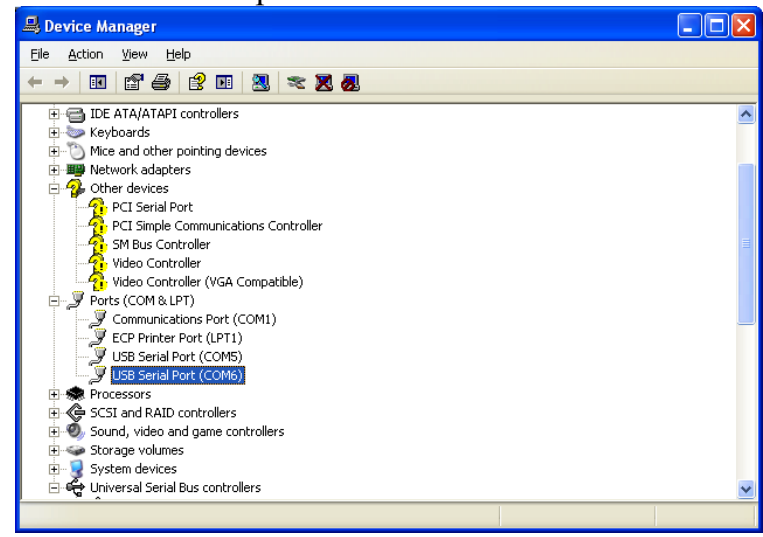

Fig. 3 Check USB Serial Port Number

4. Open the RSSI vs PDR folder and compile the Base station source code from Cygwin interface by keying in 'make micaz' (compile the program and check the errors). Then, the system will process in Fig. 4.

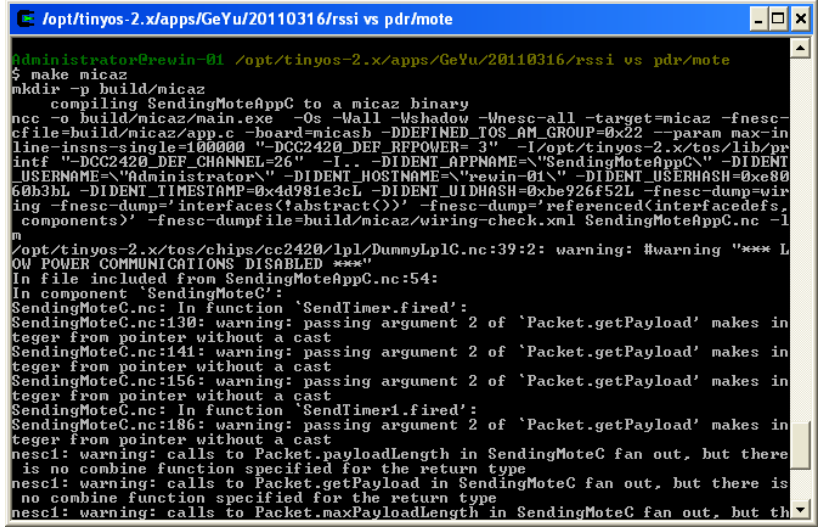

Fig. 4 Check USB Serial Port Number

5. Then the node id should be assigned to 1 each time while installing the code by using the command as Fig. 5. (Note: ttyS'x', where $\mathrm{x}=\mathrm{COM}-2$ )

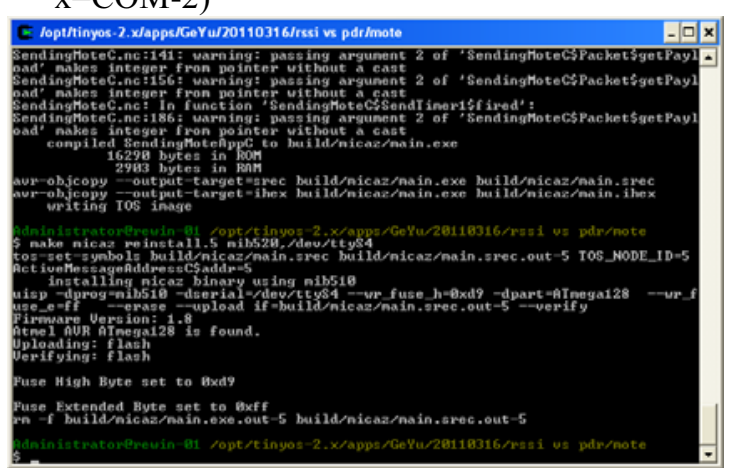

Fig. 5 Load the Code to Base Station

6. Change the transmission power in 'ApplicationDefinitions.h' in mote folder. Then repeat step 2 to step 5 with other motes, make sure the sending mote is assigned with specific node id except 1 ( 1 is only for base station).

7. Then, place them on 2 tripods separately as Fig. 6.
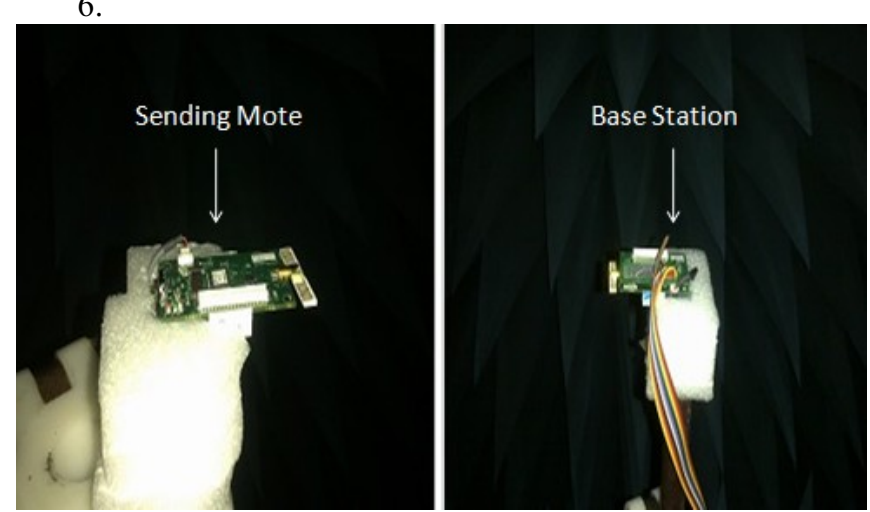

Fig. 6 Base Station \& Sending Mote

8. Then, turn on the base-station and sending mote, use the command of "java net.tinyos.tools.PrintfClient -comm serial@COM5:micaz > tx3_50cm_1.txt' in Cygwin user interface, the RSSI value per packet and total number of packet received will be recorded and saved in .txt file. The base 
station is fixed at one side of the chamber, for each transmission power it starts with the farthest distance. The sending mote is placed $250 \mathrm{~cm}$ away from the base station. Once powered on, the mote will send 1000 RSSI packets to the base station.

9. The above experiment is conducted repeatedly with different distance.

10. The main purpose is to determine the RSSI threshold value for the power control strategy which will helps to save the power supply and extend the battery usage.

\subsection{RSSI vs PDR Chamber Free-Space Result Analysis}

In this experiment, the transmission power is pre-set to 3, and different distance was engaged, and then power 7 is set to different distance between two tripods.

The distance between two tripods is fixed at $100 \mathrm{~cm}$, $200 \mathrm{~cm}$ and $180 \mathrm{~cm}$, and the result is attached in Fig. 7, Fig. 8 , and Fig. 9 respectively.

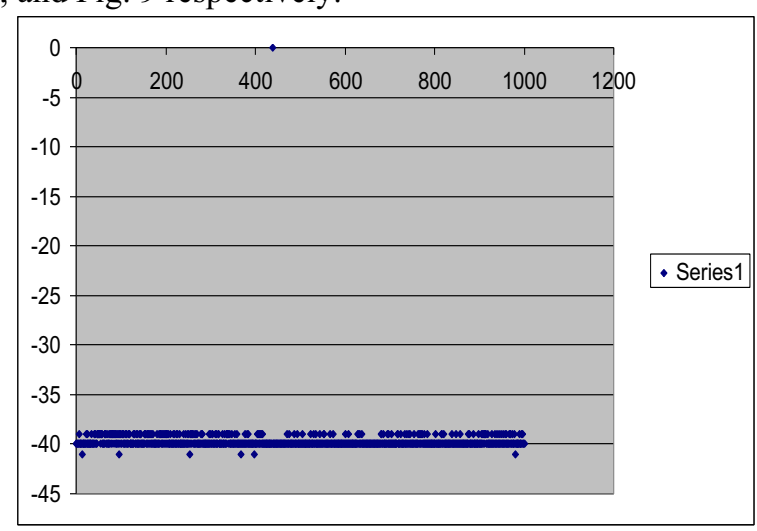

(a) RSSI Vs. Time

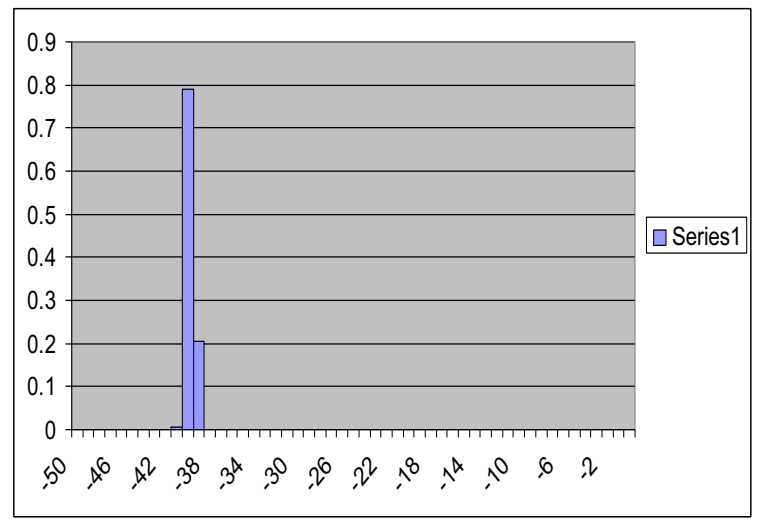

(b) RSSI Probability Distribution

Fig.7 Result @Power 3, 100cm

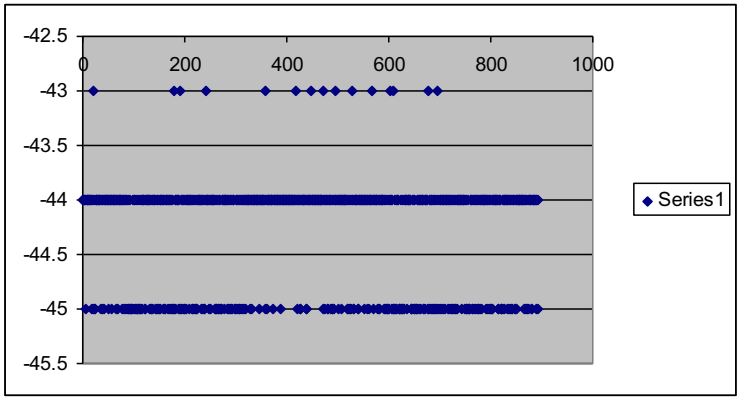

(a) RSSI Vs. Time

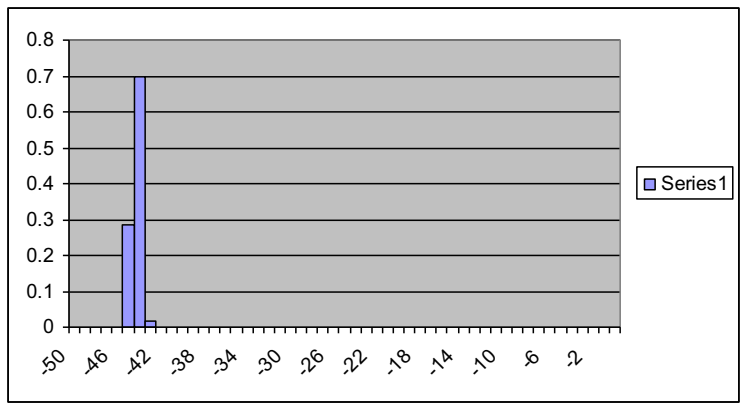

(b) RSSI Probability Distribution

Fig.8 Result @Power 3, 200cm

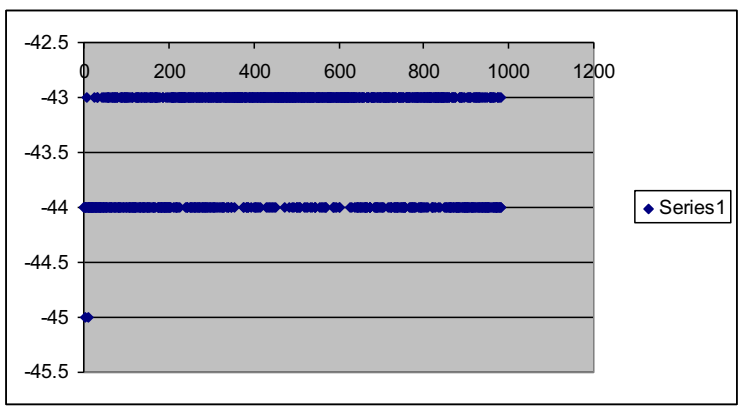

(a) RSSI Vs. Time

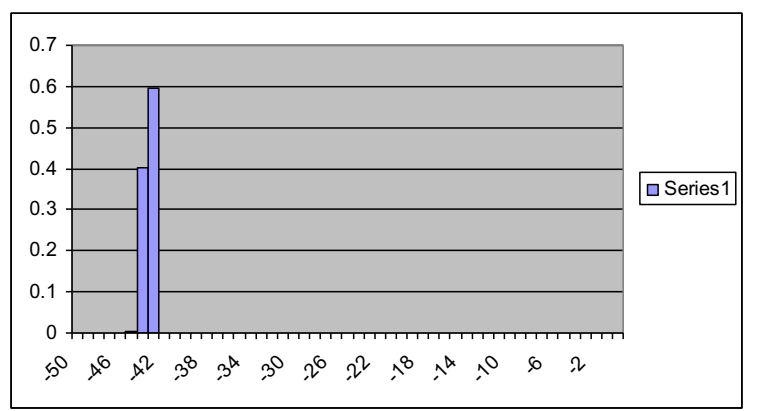

(b) RSSI Probability Distribution

Fig.9 Result @Power 3,180cm

The packet delivery ration is $100 \%$ in Fig. 7. The link performance is good, it can be seen that RSSI value is changing between ' -40 ' and ' -39 ' and majority of the RSSI values are distributed at ' -40 '. However, if the distance between two tripods is increased to $200 \mathrm{~cm}$, and the environment in chamber is clean, there will be no multipath. As shown in Fig. 8, the packet delivery ration will be $89.2 \%$, which is unacceptable for a reliable transmission. Thus the distance between two tripods is decreased to $180 \mathrm{~cm}$ as in Fig. 9. The PDR value achieves 
to $98.9 \%$ which is higher than before, and majority of the RSSI values are distributed between -43 and -44 .

Then the distance between two tripods is fixed at $300 \mathrm{~cm}$ and the transmission power is changed and pre-set to 7 . The result is as Fig. 10.

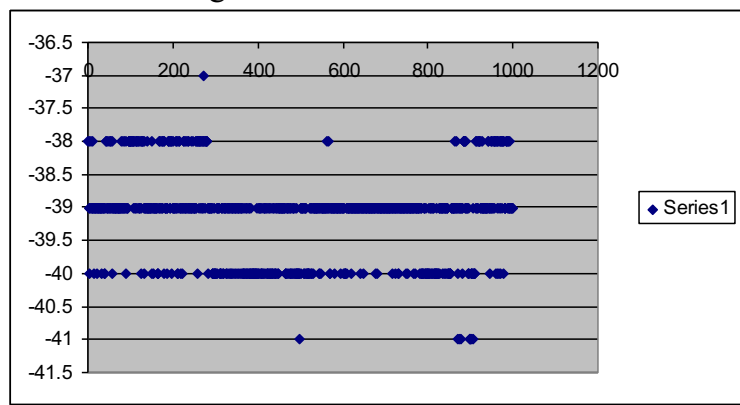

(a) RSSI Vs. Time

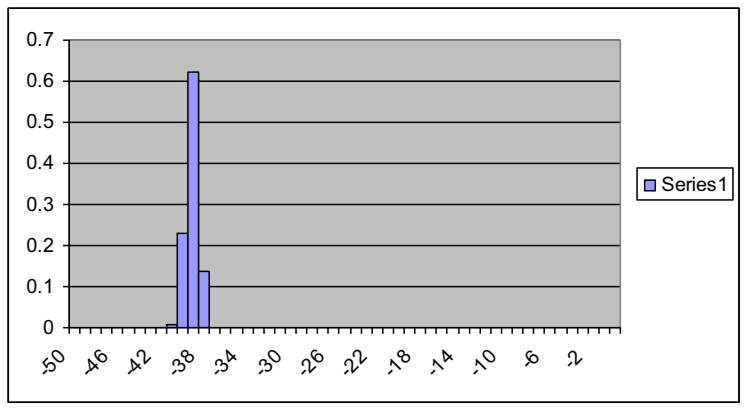

(b) RSSI Probability Distribution

Fig. 10 Result @Power 7, 300cm

From the results, it is clearly show that even the distance is $300 \mathrm{~cm}$, the PDR can still achieve to $100 \%$ with transmission power at 7 .

In conclusion, for transmission power 3, the PDR value can be higher than $95 \%$, restraining distance at $180 \mathrm{~cm}$, for RSSI value mainly distributed at ' -43 ' and ' -44 '. As a result, the threshold RSSI value is determined at ' -43 ' to keep the link transmission quality. It means that when the RSSI value of specific packet drop below ' -43 ' or the distance become larger, the mote has to increase its transmission power to a higher level to meet the PDR requirement.

\subsection{RSSI vs PDR On-Body Results Analysis}

The PDR on-body experiment result conducted in chamber will be used as reference and the standard threshold value will be determined based on the link performance. All the Micaz motes should be wear properly on each specific position on human body, basically there are 9 Micaz motes functioning as sending mote and 1 as sink which is placed at the right-hand side of waist. Each sending mote will be placed on arm, head and ankle separately as Fig. 11.

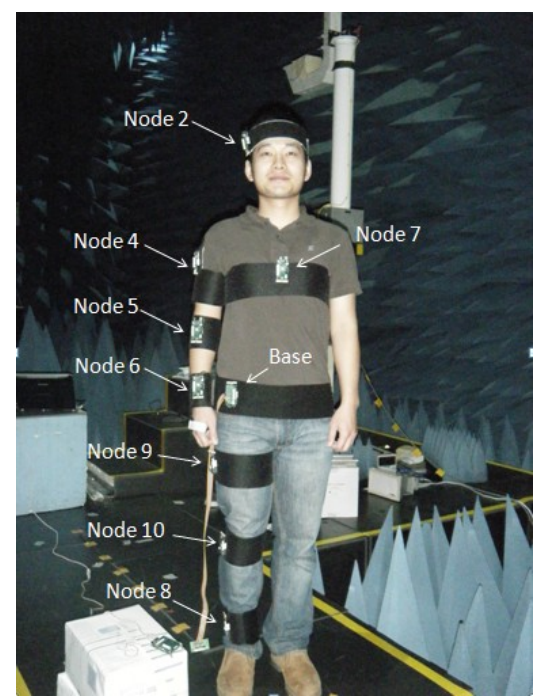

Fig. 11 Node Position

The transmission power of base-station can be any value which will not affect the performance as receiver. It will receive packet from other sending mote. By using the 'printf' function, the parent id, RSSI (radio signal strength indication) and PDR (packet delivery ratio) can be displayed from the Cygwin interface. In terms of power saving purpose, the most suitable transmission power for each specific position can be chosen.

There are basically 10 links placed properly on human body with different location. All link performance for each node has been presented and evaluated as table 1 .

Table 1. Performance Comparison of the 10 links

\begin{tabular}{|c|c|c|c|}
\hline $\begin{array}{c}\text { Node } \\
\text { Number }\end{array}$ & $\begin{array}{c}\text { Transmission } \\
\text { Power }\end{array}$ & $\begin{array}{c}\text { Majority } \\
\text { RSSI Range }\end{array}$ & PDR \\
\hline $\mathbf{5}$ & 3 & $(-40,-39)$ & $100 \%$ \\
\hline $\mathbf{6}$ & 3 & $(-18,-17)$ & $100 \%$ \\
\hline $\mathbf{9}$ & 7 & $(-36,-35)$ & $100 \%$ \\
\hline $\mathbf{1 0}$ & 7 & $(-40,-39)$ & $100 \%$ \\
\hline $\mathbf{7}$ & 11 & $(-37,-36)$ & $100 \%$ \\
\hline $\mathbf{4}$ & 11 & -44 & $96.9 \%$ \\
\hline $\mathbf{2}$ & 15 & $(-38,-37)$ & $100 \%$ \\
\hline $\mathbf{8}$ & 15 & $(-44,-42)$ & $98.9 \%$ \\
\hline
\end{tabular}

In conclusions, the distance between two nodes and transmission power are two main factors for PDR, when the distance is farther, the transmission power should be increased to meet the PDR requirement. The majority RSSI range is referred to set the RSSI threshold.

\subsection{Power Control Test}

In this section, the link performance for each predesigned RSSI value will be tested, and standard RSSI threshold value might be made based on the PDR vs RSSI results. There are several steps involved for this testing:

- Sending mote will stay at $150 \mathrm{~cm}$ away from basestation for $1 \mathrm{~min}$,

- Move it to $180 \mathrm{~cm}$ as fast as possible and stay at that point for $1 \mathrm{~min}$. 
- Move to $200 \mathrm{~cm}$ and stay for $1 \mathrm{~min}$.

- Quickly move to $220 \mathrm{~cm}$ and stay for $1 \mathrm{~min}$.

- Finally, quickly move to $250 \mathrm{~cm}$ and stay for $1 \mathrm{~min}$. From the previous RSSI vs PDR testing for transmission power 3 , the threshold value range $(-44,-37),(-43,-37)$, ($43,-36)$, and $(-43,-35)$ will be selected to run the procedure above. The comparison result is as Table 2 .

Table 2. Performance Comparison of Different RSSI Threshold Value Range

\begin{tabular}{|c|c|c|}
\hline $\begin{array}{c}\text { RSSI Threshold } \\
\text { Value Range }\end{array}$ & $\begin{array}{c}\text { Power Probability } \\
\text { Distribution }\end{array}$ & PDR \\
\hline $\mathbf{( - 4 4 , - 3 7 )}$ & $3(80 \%), 7(20 \%)$ & $69 \%$ \\
\hline $\mathbf{( - 4 3 , - 3 7 )}$ & $3(58 \%), 7(42 \%)$ & $81 \%$ \\
\hline $\mathbf{( - 4 3 , - 3 6 )}$ & $3(27 \%), 7(73 \%)$ & $97 \%$ \\
\hline $\mathbf{( - 4 3 , - 3 5 )}$ & $3(19 \%), 7(81 \%)$ & $100 \%$ \\
\hline
\end{tabular}

As shown in table $2(-44,-37)$ and $(-43,-37)$ consume less power, however, the PDR of the two modes are unacceptable, which may debase the link quality. Although the PDR value of $(-43,-35)$ is $100 \%$ for, the RSSI threshold range is a little bit broad and seems over protected. As a result, the threshold range of -43 to -36 is concluded to be the most optimal range for future experiment.

\section{Conclusions}

In this paper, the multi-hop wireless sensor network is created, which is realized by Micaz and Mib520 Board using TinyOS and Cygwin. For the hardware, the rechargeable battery is being used in order to decrease the total size of Micaz. Furthermore, the original antenna is replaced with a flat one which looks more professional and compatible with Mib520 Board. The off-the-shelf protocols and algorithms for TinyOs, RSSI and PDR, are engaged in this wireless network. Several experiments are settled to set standard RSSI threshold to allow the user to send packet with efficient power level. In the medical market, this application can be customized and superior in the great saving of hardware cost with very high accuracy to fulfil divergent requirements.

\section{Acknowledgement}

Project supported by National Basic Program of China (973 program, project number: 2011CB302402) and National Nature Science Foundation of China (No. 11171053)

\section{References}

1. CHEN Tao, LIU Jing-tai, BIN Zhi-gang. Research on the Application of Wireless Sensor Network. Automation \& Instrumentation, 2005(S1).

2. Daniel Morrison, Taran Ablitt, and Jean-Michel Redouté. Miniaturized Low-Power Wireless Sensor Interface, Sensors Journal, Vol. 15, Issue. 9, 2015, pp: 4371-4372
3. Kadir Tohma, Merve Nilay Aydın ve İpek Abasıkeleş Turgut. Improving the LEACH protocol on Wireless sensor network, $201523^{\text {rd }}$ Signal Processing and Communications Applications Conference (SIU), 16-19 May 2015, pp: 240-243

4. Wang, Rui, Zhang, Lei, Sun, Rongli, Gong, Jibing, et al. A pervasive traffic information acquisition system based on wireless sensor networks. IEEE Transactions on Intelligent Transportation Systems; Special Issue on Artificial Transportation Systems and Simulation. 2011

5. Tan X, Li J. Cooperative positioning in underwater sensor networks. IEEE Transactions on Signal Processing. 2010

6. WU Xiaona, WANG Man. Introduction of Wireless Sensor Network Operation System TinyOS. Computer and Modernization. 2011(02)

7. Cygwin user guide. www.cygwin.com .

8. WU Gui-ping, YU Hui-qun, FAN Gui-sheng. A Sensor Network Allocation Algorithm based on RSSI. Journal of East China University of Science and Technology (Natural Science Edition). 2013(05)

9. Lee Yang Sun,Lee Ji Min,Yeo Sang Soo.et al.A study on the performance of wireless localization system based on AoA in WSN. 2011 Third International Conference on Intelli Kent Networking and Collaborative Systems. 2011 